Relations industrielles

Industrial Relations

\title{
Morin, Fernand. Rapports collectifs du travail
}

\section{Jean-Yves Brière}

Volume 47, numéro 4, 1992

URI : https://id.erudit.org/iderudit/050816ar

DOI : https://doi.org/10.7202/050816ar

Aller au sommaire du numéro

Éditeur(s)

Département des relations industrielles de l'Université Laval

ISSN

0034-379X (imprimé)

1703-8138 (numérique)

Découvrir la revue

Citer ce compte rendu

Brière, J.-Y. (1992). Compte rendu de [Morin, Fernand. Rapports collectifs du travail]. Relations industrielles / Industrial Relations, 47(4), 789-792.

https://doi.org/10.7202/050816ar

Tous droits réservés (C) Département des relations industrielles de l'Université Laval, 1992
Ce document est protégé par la loi sur le droit d’auteur. L’utilisation des services d'Érudit (y compris la reproduction) est assujettie à sa politique d'utilisation que vous pouvez consulter en ligne.

https://apropos.erudit.org/fr/usagers/politique-dutilisation/ 


\section{RECENSIONS BOOK REVIEWS}

Rapports collectifs du travail, par Fernand Morin, Montréal, Les Éditions Thémis, 1992, 831 p., ISBN 2-920376-97-7

Le droit comme la plupart des sciences humaines et même des sciences dites «exactes» est en proie à la frénésie de l'information. Le praticien, l'enseignant et l'étudiant en droit du travail sont littéralement ensevelis sous une masse d'information que je qualifierais d'être à l'état brut. Nous avons facilement accès à des centaines de décisions par année, les banques de données pullulent et plusieurs ouvrages colligent et compilent le fruit du labeur des différents décideurs. A certains égards, nous pourrions même parler «d'idolâtrie moderne de l'information" (Georges Steiner, Réelles présences, les arts du sens, Paris, Gallimard, 1991, 286 pages). Cependant, l'accès à l'information ne doit pas être une panacée qui nous éloigne de la nécessité de la réflexion théorique. A cet égard, il est important de souligner la parution récente de la dernière version de l'ouvrage du professeur Morin portant sur les rapports collectifs du travail, car il constitue une excellente synthèse et il contient de nombreux éléments de réflexion théorique sur cette province du droit que sont les rapports collectifs du travail.

Comme le souligne l'auteur dans l'avant-propos, cette seconde édition était devenue nécessaire parce que «le droit du travail évolue si rapidement et la mutation des données socio-économiques sur lesquelles it repose est parfois si drastique» qu'il a fallu reprendre l'ouvrage. Fait à signaler, il ne s'agit pas d'une simple mise à jour ou d'un peaufinage de l'oeuvre originale. En effet, le livre comporte quelque deux cents pages de plus que le premier. Ces ajouts sont consacrés d'abord aux amendements qui furent apportés au Code du travail en 1983 et également à la présence sans cesse croissante de l'État dans les relations du travail. Finalement, l'auteur fait largement écho des libertés publiques fondamentales garanties aux Chartes canadienne et québécoise des droits et libertés. L'évolution de l'état du droit sur ces sujets justifiait largement le labeur du professeur Morin.

Le plan retenu par l'auteur comporte six chapitres: 1. Données fondamentales; 2. Aperçu du droit syndical; 3. L'accréditation; 4. Négociation collective des conditions de travail; 5 . La convention collective de réglementation du travail et son administration; 6 . Les services et les secteurs publics.

Le corps du texte est suivi de certaines annexes et d'index (jugements cités, lois citées, index analytique) qui facilitent grandement la recherche. Avant d'aborder chacun des chapitres, soulignons que l'introduction, particulièrement soignée, permet de ufaire précéder les analyses techniques et particulières de notions générales et de vues d'ensemble». Ainsi, l'auteur nous présente les causes historiques du droit du travail. II circonscrit les tenants et aboutissants 
du droit du travail teis, la définition de ce droit et ses traits caractéristiques. Finalement, il nous soumet la pièce législative majeure, soit le Code du travail.

Le chapitre I (Données fondamentales) introduit les trois principaux acteurs du droit du travail: le salarié, l'employeur et l'État. Dans ce chapitre sont traitées, entre autres, les questions suivantes: $-Y$ a-t-il encore un contrat de travail lorsque le salarié est assujetti à une convention collective? - Comment distinguer l'employeur, l'entreprise et l'établissement? - Faudrait-il identifier l'employeur par la maison-mère, la société de portefeuille, le trust, le cartel ou la multinationale qui détient le contrôle et qui, en quelque sorte, est le maître de sa destinée? - Quel est le statut de l'employeur pour la tenue des rapports collectifs? Quels sont les moyens mis en place par l'État pour intervenir dans les rapports collectifs du travail? etc... L'auteur répond à toutes ces questions en précisant la place de chacun des acteurs dans ce parfois difficile ménage à trois.

Le chapitre II (Aperçu du droit syndical) se cristallise autour de deux thèmes. D'abord, la liberté syndicale, c'est-à-dire l'affirmation de ce droit fondamental et les modalités garantissant son exercice et ce, tant au niveau individuel que collectif. Á l'aide d'une approche historique, l'auteur cerne cette liberté fondamentale et en détermine l'étendue. De plus, l'auteur s'attarde particulièrement sur la protection accordée par les Chartes car dans ce domaine, le chemin tracé par les tribunaux de droit commun est parfois sinueux et il est important de circonscrire la portée réelle de la garantie constitutionnelle qu'offre la Charte canadienne.

Le deuxième thème de ce chapitre est celui des aspects juridiques du gouvernement syndical. Cette section vise à déterminer les conditions que doivent respecter les salariés pour créer un syndicat et participer démocratiquement à sa gestion et à son administration. Pour ce faire, l'auteur détermine d'abord le statut juridique reconnu au syndicat et dans un second volet, il explique comment les syndiqués peuvent vraiment et librement participer au processus décisionnel de leur syndicat. Plusieurs pages sont consacrées au devoir de juste et loyale représentation du syndicat. Sur cette importante question, l'étude de Fernand Morin est des plus complètes et fort pertinente. Non seulement expose-t-elle l'état du droit et de la jurisprudence, mais elle souligne également les multiples défaillances du régime.

Le chapitre III porte sur l'accréditation. Monsieur Morin nous souligne qu'un syndicat ne peut imposer sa présence à l'employeur. II se doit de franchir une étape préliminaire, à savoir, se soumettre au "contrôle de qualité" de l'État, en somme, à la vérification de son caractère représentatif. Cet examen s'effectue par le biais de l'accréditation. Comme le précise l'auteur: "Par ses multiples facettes historiques, politiques et techniques, l'accréditation constitue l'enclume sur laquelle est façonné le particularisme même de notre régime des rapports collectifs». Face à l'importance du sujet, il nous présente un chapitre très fouillé. La procédure d'accréditation est littéralement disséquée. Ainsi, sont traités: - la requête; - le requérant; - le moment utile; - la démarche à suivre; l'administration; - l'unité appropriée; - le constat de représentativité; - les procédures complémentaires ou incidences à l'accréditation. 
Finalement, le chapitre se termine sur les effets de l'accréditation. Dans cette dernière section une part importante est consacrée aux articles 45 et $46 \mathrm{du}$ Code du travail, soit l'aliénation ou la concession totale ou partielle d'une entreprise. Cette section a le mérite d'exposer simplement et clairement l'état du droit sur un sujet des plus controversés en droit du travail. Le professeur Morin analyse largement l'arrêt de la Cour suprême du Canada dans l'affaire Bibeault, non seulement pour l'expliquer mais également pour en montrer les faiblesses et les limites.

Le chapitre IV (Négociation collective des conditions de travail) est l'aboutissement du processus de l'accréditation, soit le droit qui est accordé au syndicat d'entamer la négociation collective des conditions de travail. Le chapitre est divisé en trois parties. Dans un premier temps, l'auteur aborde la négociation directe, soit les modalités d'exercices de la négociation collective. Dans un deuxième temps, la négociation est décrite dans son mode conflictuel soit la négociation sous pression. La grève y est analysée en faits et en droit. Les décisions récentes de la Cour suprême du Canada portant sur le droit de grève et la Charte canadienne y sont discutées. Dans un troisième temps, l'auteur aborde l'élaboration des conditions de travail par un tiers et ce, qu'il soit volontairement choisi par les parties ou imposé par la loi. L'arbitrage comme mode de détermination des conditions de travail est décrit, analysé et commenté.

Le chapitre $V$ (la convention collective de réglementation du travail et son administration) traite de la convention collective et de l'arbitrage de griefs. Le chapitre comprend d'abord une analyse des aspects juridiques de la convention collective et ce, en mettant en relief ses traits caractéristiques. De plus, ses conditions d'existence propres sont décrites ainsi que son possible contenu. L'auteur termine la première section du chapitre en examinant les effets de la convention et en répondant à la question de savoir quelle est la nature juridique de la convention collective. La deuxième section porte sur le contentieux qui résulte de l'administration de la convention collective. Le processus d'arbitrage y est littéralement anatomisé. L'évolution des règles et de la fonction de l'arbitrage et de l'arbitre sont étudiées. Le processus arbitral (le grief, les parties, les rencontres préalables, la nomination de l'arbitre, son statut et ses pouvoirs, l'enquête, la preuve) y est décrit dans son aspect dynamique. Finalement, le chapitre prend fin sur la sentence arbitrale, ses effets et son possible contrôle judiciaire.

Le chapitre VI (les services et les secteurs publics) nous amène dans les dédales des services et des secteurs publics. Quatre mots furent ajoutés à la définition d'employeur en 1965 ("y compris sa Majesté") pour assujettir l'État au Code du travail. La réalité imposa que l'on complexifie le cadre juridique du régime. Ainsi, le législateur est intervenu à tous les deux ou trois ans pour fixer de nouvelles modalités au régime. La vue d'ensemble qui se dégage de la présentation du chapitre est fort utile puisqu'elle permet de faire le point sur l'état des règles de droit aménageant les rapports collectifs publics. D'abord certaines généralités sont dégagées: un bref rappel historique et quelques définitions. Suit une description des organes mis en place pour assurer la bonne marche du système. On y présente les parties qui sont les interlocuteurs à la négociation. Troisièmement, on aborde la négociation et les règles relatives au maintien des services essentiels. Finalement, on analyse la convention collective et 
l'arbitrage de griefs. Peu d'auteurs se sont penchés sur les particularismes de ce régime qui, en quelque sorte, influence directement ou à tout le moins indirectement le quotidien de tous les Québécois. De ce fait, le texte revêt une importance particulière. De plus, il soulève de façon claire et précise, les contradictions du système tel le double rôle de l'État qui est à la fois législateur et employeur. Finalement, l'auteur souhaite une réforme qui permettrait un réel retour aux sources, "c'est-à-dire aux établissements et institutions, aux syndicats locaux et aux gestionnaires mais nullement par voie de délégation condescendante des centrales syndicales et du Conseil du trésor ". II plaide pour un «changement réel d'une praxis, délaissant ainsi quelque stéréotypes révolus».

L'oeuvre dans son ensemble constitue un outil de travail indispensable pour tous ceux, praticiens, professeurs et étudiants, qui s'intéressent à titre de profanes ou de spécialistes aux rapports collectifs du travail. Tous y trouveront leur compte. De plus, et c'est peut-être le plus important à mes yeux, le professeur Morin sait soulever les interrogations et les remises en question qui, par les mutations qu'elles opèrent, suscitent chez le lecteur une réflexion lui permettant de cheminer. Cette démarche me semble beaucoup plus utile que celle de certains auteurs qui prétendent répondre à toutes les questions du lecteur.

Jean-Yves BriérE

Montréal

Industrial Relations in Canadian Industries, by Richard P. Chaykowski and Anil Verma, eds, Toronto, Dryden (Holt, Rinehart and Winston of Canada), 1992, 491 p., ISBN 0-03-922877-0

L'objectif de ce volume est de tenter de cerner les changements survenus dans les relations industrielies au Canada au cours des dix dernières années afin de déterminer si les pratiques en vigueur continueront de s'apparenter à ce que les auteurs appellent le "modèle traditionnel» qui a prédominé de la deuxième Grande Guerre jusqu'au début des années 1980 ou si, au contraire, on peut déceler dans la panoplie de pratiques nouvelles qui se sont développées récemment des indices permettant de conclure à l'apparition d'un nouveau modèle de relations industrielles au Canada. Ce livre fait donc écho à une analyse similaire effectuée il y a quelques années aux États-Unis et qui avait donné lieu à la publication du "best seller» The Transformation of American Industrial Relations, par Kochan, Katz et McKersie (New York, Basic Books, 1986). La préface du volume est d'ailleurs écrite par Thomas Kochan.

Comme dans l'étude portant sur les États-Unis, les deux co-éditeurs s'inspirent de l'approche dite «des choix stratégiques» tout en mettant à contribution l'approche systémique traditionnelle. Cependant, contrairement à The Transformation of American Industrial Relations où les trois auteurs avaient procédé à une étude globale portant sur l'ensemble de l'économie américaine (qui s'appuyait par contre sur les résultats des très nombreuses recherches empiriques auxquelles ils avaient déjà participé), Verma et Chaykowski ont, pour 